\title{
Histological Assessment of Bone Regeneration by Octacalcium Phosphate and Bone Matrix Gelatin Composites in a Rat Mandibular Defect Model
}

Fereydoon Sargolzaei Aval ${ }^{1}$, Eshagh Ali Saberi ${ }^{2}$, Mohammad Reza Arab ${ }^{1}$, Narjes Sargolzaei ${ }^{3}$, Esmaeel Zare ${ }^{2}$, Heshmatallah shahraki ${ }^{2}$, Forough Sargolzaeiaval ${ }^{4}$, Maryam Arab ${ }^{1}$

1 - Cellular and Molecular Research Center \& Department of Anatomical Sciences, School of Medicine, Zahedan University of Medical Sciences, Zahedan, Iran.

2 - Department of Endodontics, School of Dentistry, Zahedan University of Medical Sciences, Zahedan, Iran.

3 - Department of Community Medicine, School of Medicine, Zahedan University of Medical Sciences, Zahedan, Iran.

4 - Department of Pathology, University of Washington, Seattle, Washington, USA.

Address correspondence to Fereydoon Sargolzaei Aval, Dept. of Anatomical Sciences, School of Medicine, Zahedan University of Medical Sciences, Zahedan, Iran.

Tel: $+9854-33295722 \quad$ Fax: $+9854-33295728$

E-mail: fsargolzaei@yahoo.com

Conflict of interest: None declared

Running Title: Bone regeneration Induced by Octacalcium Phosphate and Bone Matrix Gelatin 


\begin{abstract}
Objective: Regeneration of bone defects remains a challenge for maxillofacial and reparative surgeons. The purpose of this histological study was to assess the osteogenic potential of octacalcium phosphate (OCP) and bone matrix gelatin (BMG) alone and in combination in artificially created mandibular bone defects in rats. The quality of the newly formed bone was also evaluated.
\end{abstract}

Methods: Thirty-six male Sprague Dawley rats (6-8 weeks old with 120-150 g weight) were randomly divided into four groups. Defects ( $3 \mathrm{~mm}$ in diameter and $2 \mathrm{~mm}$ in depth) were created in the mandible of rats and filled with $6 \mathrm{mg}$ of $\mathrm{OCP}, \mathrm{BMG}$ or a combination of both (1/4 ratio), respectively. Defects were left unfilled in the control group. To assess osteoinduction and bone regeneration and determine the quality of the newly formed bone, tissue specimens were harvested at seven, 14, and 21 days post-implantation. The specimens were processed, stained with hematoxylin and eosin (H\&E) and histologically analyzed under light microscopy.

Results: In the experimental groups, new bone formation was initiated at the margins of defects from seventh day after implantation. At the end of the study period, the amount of the newly formed bone increased and the bone was relatively mature. Osteoinduction and new bone formation were greater in $\mathrm{OCP} / \mathrm{BMG}$ group. In the control group, slight amount of new bone had been formed at the defect margins (next to host bone) on day 21 .

Conclusion: Combination of OCP/BMG may serve as an optimal biomaterial for treatment of mandibular bone defects.

Keywords: Octacalcium phosphate, Bone matrix gelatin, Osteogenesis 


\section{Introduction}

Regeneration of bone defects caused by trauma, infection, or resection of bone tumors remains a challenge in orthopedic and maxillofacial reconstructive surgeries. Autogenous bone grafts are the gold standard of treatment for small defects. However, due to limited quantity, they cannot be used for large defects and biocompatible bone substitutes may be applied instead [1, 2]. A group of synthetic bone substitutes have osteoinductive properties[3-5] while another group of these materials are osteoconductive and serve as a carrier for preservation and gradual release of bone morphogenetic proteins(BMPs) [6,7].

Calcium phosphate derivatives are among the most commonly used synthetic biomaterials for hard tissue regeneration [8]. These materials are also categorized into different groups depending on the ratio of calcium/phosphate in their composition. They highly resemble the natural mineral content of bone and tooth in terms of physical and chemical properties [9]. Different compositions of calcium phosphate materials have variable potential for regeneration of bone defects and some of them only serve as a carrier for osteoconductive proteins [7, 10, 11]. Some others are both osteoconductive and osteoinductive when placed adjacent to bone defects [12, 13]. Octacalcium phosphate (OCP) has both osteoconductive and osteoinductive properties and is believed to be the direct precursor of hydroxyapatite. This material, compared to other calcium phosphate derivatives, has higher potential for both osteoinduction and osteoconduction. It is gradually absorbed and replaced by the newly formed bone $[14,15]$. Based on the literature, OCP has shown promising results for healing bone defects when used alone [16] or in combination with biomaterials such as Bone Matrix Gelatin (BMG) or transforming growth factor beta (TGFß) $[17,18]$.

On the other hand, successful healing of bone defects reported after BMG implantation has encouraged researchers to use this material as a synthetic alternative to autogenous bone grafts 
$[19,20]$. Bone matrix gelatin contains type I collagen as well as a high protein content including Bone Morphogenetic Proteins (BMPs), which induce the differentiation of mesenchymal cells to osteoblasts at the site of bone defects [21]. The interaction of BMPs with most calcium phosphate derivatives has yielded favorable results in clinical applications [17,22, 23].

We aimed to assess the osteogenic potential of BMG and OCP implanted alone or in combination in mandibular bone defects of rats. The quality of the newly formed bone was also evaluated.

\section{Materials and Methods}

\section{A. Preparation of materials:}

Synthetic OCP was prepared following the methods previously described [24, 25]. The sieved granules (particle size of 300-500 $\mu \mathrm{m}$ ) of OCP, obtained from dried OCP, were sterilized by heating at $120^{\circ} \mathrm{C}$ for 2 hours. A previous study showed that such heating does not affect physical properties such as the crystalline structure or specific surface area of OCP granules [26, 27]. Bone matrix gelatin (BMG) was prepared using the method previously described by urist et al. [28] which was modified by the method of Wei-Qi Yan et al. [3]. Briefly, diaphyseal shafts of femora and tibiae from 6-week-old male Sprague dawley rats were cut into chips. Liquid nitrogen was used to freeze bone shafts after their removal and while they were being cut into chips to avoid possible denaturation of proteins, the bone chips were extracted in chloroform-methanol (1:1) for 4 hours at $25^{\circ} \mathrm{C}$, demineralized in $0.6 \mathrm{~N}$ of hydrochloric acid at $4{ }^{\circ} \mathrm{C}$ for 72 hours, and gelatinized in $6 \mathrm{M}$ of lithium chloride at $2{ }^{\circ} \mathrm{C}$ for 24 hours. The bone chips then were auto digested at $37^{\circ} \mathrm{C}$ for 48 hours in phosphate buffer (pH 7.4) with $10 \mathrm{mM}$ of sodium azide and 5 $\mathrm{mM}$ of iodoacetic acid as a protease inhibitor. The bone chips then were pulverized with a sample chamber and sifted. Particles sized 75-500 $\mu \mathrm{m}$ were collected by the testing sieve, lyophilized, sterilized in ethylene

\section{B. Sample size:}


This experimental study was conducted on 36 adult (6-8 weeks old) male Sprague-Dawley rats with a mean weight of 120-150 g. The animals were obtained from the Animal Research Center of Zahedan University of Medical Sciences. The animals were randomly divided into three experimental (OCP, BMG, and $\mathrm{OCP} / \mathrm{BMG}$ ) groups and one control group and kept in standard conditions with light/dark cycles of equal duration. The principles of laboratory animal care, as well as national low, were followed. All procedures were approved by the Ethics Committee for Animal Experiments of Zahedan University of Medical Sciences.

\section{Surgical procedure and implantation of materials:}

Animals were randomly divided into four groups as described above and anesthetized by intraperitoneal injection of $60 \mathrm{mg} / \mathrm{kg}$ ketamine hydrochloride (Ketalar, Trustech Pharma Care, Bayern, Germany) and $20 \mathrm{mg} / \mathrm{mL}$ xylazine (Pantex Holland B.V., Duizel, Netherland) in 2/1 ratio. Diethyl ether was used for anesthesia maintenance. After the induction of general anesthesia, the animals were fixed on the operating table in a supine position. The respective area on the body of the mandible was shaved and disinfected using 10\% Betadine (Toliddarou, Tehran, Iran). During the operation, corneal dryness was prevented using Bactimide ${ }^{\circledR}$ eye drop (Toliddarou, Tehran, Iran). Using a sterile surgical scalpel, a $1.5 \mathrm{~cm}$ incision was made on both sides of the mandible and a full-thickness periosteal flap was elevated. Using a dental drill, a defect measuring $3 \mathrm{~mm}$ in diameter and $2 \mathrm{~mm}$ in depth was drilled in the mandible close to the alveolar crest (in-between the first molar and canine teeth) under copious irrigation with cold saline solution.

In the first experimental group, $6 \mathrm{mg}$ of OCP (previously prepared and packed) was implanted at the site. In the second experimental group, $6 \mathrm{mg}$ of BMG and in the third experimental group, 6 mg of OCP/BMG with a $1 / 4$ ratio were implanted in defects. After implantation of the materials, the defects in all experimental groups were covered with Surgicel (Altaylar, Medical, Ankara, 
Turkey) to prevent the spread of implanted particles. Defects were left empty in the control group and were only covered with Surgicel. The skin and the underlying connective tissue at the surgical site were sutured in two layers using a $4 / 0$ absorbable chromic suture (catgut, Wei Gao Group Kanglida Medical Products Co, LTD. Heze, China) and disinfected. After completion of the operation and recovery of the rats from anesthesia, they were transferred to hygienic cages and kept there until sacrifice at the end of the time table.

\section{Harvesting tissue samples:}

In all experimental and control groups, tissue samples were harvested at seven, 14, and 21 days (from six rats sacrificed at each time point) to prepare histological sections. At the mentioned time points, general anesthesia was induced by intraperitoneal injection of ketamine hydrochloride. The chest was opened and 10\% buffered formalin (fixative) was perfused through the heart to achieve in situ fixation of tissues. The respective area along with a margin of host bone was resected and stored in $10 \%$ buffered formalin at room temperature for one week for complete fixation. Next, the tissue specimens were rinsed with cold distilled water for several times and decalcified by immersion in a decalcifying solution containing $10 \%$ formic acid, $2.9 \%$ citric acid, and $1.8 \%$ trisodium citrate dihydrate at room temperature for four weeks [14]. The tissue specimens were routinely prepared and $5 \mu$ serial sections were made from paraffinembedded tissue blocks. The desired sections were subjected to H \& E staining for evaluation under light microscopy (Zeiss, Carl Zeiss Microscopy GmbH. Goettingen, Germany).

\section{E. Histological evaluation:}

Histological analysis of stained specimens was done blindly under a light microscope by a histologist who was blinded to the type of bone substitutes used. Photomicrographs were taken using a photomicroscope (Leica DM 500, Leica Microsystems, GmbH. Wetzler, Germany) at seven, 14 , and 21 days of experiment. 


\section{Results}

Osteogenesis in all groups was evaluated under a light microscope at seven, 14 and 21 days following implantation of bone substitutes. The results were as follows:

\section{Control group:}

First week (seven days): Defects had been filled with hyper-cellular, vascular connective tissue with a regular and completely defined host bone margin. The hyper-cellular connective tissue along with desmocytes present at the site of defects was among the most prominent histological findings in the first week (Figure 1a).

Second week (14 days): Hyper-cellular, vascular connective tissue had completely filled the defects. Local invasion of the connective tissue into some areas of the defect margin (host bone) was clearly seen. The entire defect margin (adjacent to the host bone) had been covered by basophilic osteoblast-like cells (Figure 1b).

Third week (21 days): Greater penetration of more organized connective tissue into the surrounding host bone was noted.A cluster of newly formed bone matrix was observed near the margin of the bone defects. Other characteristics of the connective tissue were similar to those reported in the previous time points except for higher cellularity at this time (Figure 1c).

OCP group:

First week (seven days): The amount of the newly formed bone around the implanted particles was almost similar to the amount of implanted particles. Other characteristics of the connective tissue were similar to those mentioned for the control group, which indicates the differentiation potential of mesenchymal cells around the implanted particles (Figure 2a). 
Second week (14 days): Osteoinduction and osteoblastic differentiation at the site of defect and around the implanted particles along with complete organization of bone tissue at the defect margin adjacent to the host bone were noted. A basophilic line separated the newly formed bone from the host bone at the margins. Penetration of connective tissue into the newly formed bone around the implanted particles at the defect margins clearly indicated the osteogenic potential of these particles starting from the periphery of defect towards the newly formed bone (Figure 2b).

Third week (21 days): Induction of bone formation and complete organization of the newly formed bone at the defect site were noted. The newly formed bone had extended from the margins of the defect towards the center. The newly formed bone had been penetrated by the connective tissue at the center of defect. This pattern indicated induction of osteogenesis and new bone formation starting at the periphery of the defect (adjacent to the host bone) and extending towards the deeper areas of the defect (Figure 2c). Multi-nuclear pre-osteoclasts adjacent to the implanted particles were also seen. These cells had acidophilic cytoplasm at the tip and a basophilic base (site of accumulation of nuclei), which were typical characteristics of osteoclasts. These cells probably open the path by absorbing the implanted particles and allow for the penetration of pre-osteoblasts and initiation of bone matrix deposition (Figure 2d).

BMG group:

First week (seven days): The implanted BMG particles were seen as distinct eosinophilic masses at the defect area. The defect margin and the central area (full of BMG particles of variable sizes) were clearly visible. The spaces in-between BMG particles had been filled with a relatively dense fibrous connective tissue. Cellularity of the connective tissue was greater adjacent to the BMG particles compared to farther areas. The staining of cellular masses was also different at different sites. Connective tissue had penetrated into the matrix porosities in-between BMG particles, dividing each mass into smaller pieces. These gaps appeared to be indicative of initiation of cell 
differentiation within each particle because the orientation of these cells in BMG gaps was similar to their orientation on the surface of primary particles (Figure 3a). In one specimen in this group, osteoblasts along with deposited osteoid material were clearly visible at the margins (host bone) and a basophilic line clearly separated the osteoid material from the host bone (Figure 3b).

Second week (14 days): Evidence of inflammatory infiltration still existed. Defect margins were clearly visible. The connective tissue had become more organized and filled the gaps in-between BMG particles. Some implanted particles had been covered with differentiated osteoblasts; these osteoblasts had clearly adhered to these particles. Smaller particles had allowed better invasion of blood vessels and connective tissue into them showing evident signs of cell differentiation. The osteogenic potential was more evident in smaller particles compared to large ones with no such penetration. Signs of intramembranous ossification were seen close to the defect margin and adjacent to the host bone. This pattern was less visible at the center of the defect. A basophilic line separated the newly formed bone from the host bone (Figure 3c). In some specimens in this group, chondroblast-like cells were seen at the center of defect and over some of the BMG particles and a cartilaginous-like tissue was noted in this area (figure not shown).

Third week (21 days): Similar to the second week, a distinct basophilic line separated the newly formed bone from the host bone at the margins of defects; the only difference was that the newly formed bone highly resembled the host bone and had filled a larger part of the defect to a greater depth. Primary osteon pattern was clearly visible in this group and initiation of the remodeling of bone trabecula was clearly seen (Figure 3d). At the central areas, the connective tissue was well organized and most BMG particles had been phagocytosed by the multinucleated giant cells. In terms of staining properties, the newly formed bone showed high resemblance to the host bone (Figure 3e). 


\section{OCP/BMG group:}

First week (seven days): Signs of inflammation along with cell differentiation were evident over the implanted particles (both OCP and BMG). The gaps in-between the particles had been filled with a relatively dense connective tissue full of fibrous components. Signs of cellular attachment were evident over the BMG particles. A porous pattern of the particles was seen over the entire defect area (Figure 4a).

Second week (14 days): The defect site, containing the implanted particles, had been clearly filled with a dense connective tissue and evidence of cell adhesion and differentiation was noted around the implanted particles. The gaps in-between the implanted particles had been filled with loose connective tissue and large basophilic cells very similar to giant cells (Figure 4b). In some specimens in this group, induction of bone formation was significantly greater at the margins of the defect adjacent to the host bone compared to the central areas. Moreover, penetration of mesenchymal cells into BMG particles was greater than into OCP particles; consequently, induction of osteogenesis was greater around $\mathrm{BMG}$ particles as well. Infiltration was greater at the central parts of the defects compared to marginal areas (Figure 4c).

Third week (21 days): Similar to previous time points, areas at the periphery of the defect and close to host bone showed signs of better osteoblastic differentiation, initiation of osteogenesis and deposition of bone matrix compared to the central areas. The morphology and characteristics of the connective tissue filling the gaps between the implanted particles were similar to the findings at earlier time points. The only difference was that higher cell differentiation was noted adjacent to OCP particles and penetration of connective tissue into BMG particles had increased. From staining point of view, the organized newly formed bone showed an eosinophilic pattern similar of ECM to the host bone (Figure 4d). 


\section{Discussion}

The efficacy of synthetic bone substitutes alone or in combination for bone regeneration has been evaluated in many previous studies $[1,14,21]$. The current study sought to assess the osteogenic potential of BMG and OCP implanted alone and in combination in artificially created bone defects in the mandible of rats. The osteoinductive and osteoconductive properties of these materials were investigated as well. In other words, we tried to combine the osteoinductive properties of BMG with the osteoinductive and osteoconductive properties of OCP to benefit from their synergistic properties and enhance mandibular bone regeneration. The results showed that implantation of OCP at the site of bone defects induced intramembranous (woven) bone formation. Inflammatory cell infiltrates were noted around the OCP particles, surrounded by an organic matrix. This indicated host response to OCP. Kamakura and colleagues reported similar reaction to OCP implantation at the site of defects created on the parietal bone [16]. At three weeks, bone trabecula with an irregular pattern were seen at the defect site in the OCP group in our study. Osteoblasts were noted on the surface and osteocytes were clearly seen at the center of bone trabecula, which were indicative of the organization of the newly formed bone trabecula and their remodeling. Most researchers have reported this finding eight weeks following OCP implantation $[25,29]$.

The mechanism of osteoinduction by the implanted OCP particles at the site of mandibular bone defects has yet to be fully elucidated and their biological properties responsible for induction and progression of bone formation are not known either. Suzuki and co-workers believe that the glycoconjugates present in the tissue accumulated over and around the OCP particles, play a role in osteogenesis [25]. In another study, Suzuki and colleagues [14] stated that the biological properties and the role of OCP in stimulating bone formation are similar to those of TGF- $\beta$. He added that bioactive factors such as TGF- $\beta$ are present in the tissues or tissue fluids accumulated around and over the OCP particles and these factors may be responsible for osteoblastic 
differentiation, new bone formation and chemotaxis of osteoclasts for bone remodeling. Sasano and co-workers [30] reported that the biological properties of OCP in terms of osteoinduction and osteoconduction were comparable to those of prostaglandin E1. They explained that prostaglandin E1 accumulates in the tissues and tissue fluids surrounding the OCP particles. Release of prostaglandin E1 may continue for as long as the OCP particles are present at the site, enhancing osteoblastic differentiation. Our results showed that the first step for osteogenesis is induced by the host bone at the most peripheral areas of defect and then gradually extends towards the center.

We found that signs of intramembranous bone formation were evident at the defect margins in the BMG group in the second week following implantation. The timing of new bone formation in this group was similar to the findings of a previous study [31]. On the other hand, appearance of osteoblasts and formation of bone tissue occurred sooner than the cartilaginous tissue and the amount of the new bone formed directly due to osteoblastic differentiation was greater than the amount of bone formed by replacement of residual spaces following destruction and re-absorption of cartilaginous tissue. Thus, it may be stated that cartilaginous tissue played an insignificant role in osteogenesis in the defects, and formation of new bone was mainly the direct result of osteoblastic differentiation. This finding was in agreement with the results of Wang and colleagues [32], who reported intramembranous ossification following implantation of demineralized bone matrix (DBM) in the cranial region. Thus, it can be concluded that in contrast to endochondral ossification, which occurs following implantation of DBM subcutaneously, intramuscularly or in defects of long bones, implantation of BMG particles in mandibular bone defects of rat results in direct proliferation and differentiation of mesenchymal cells to osteoblasts and consequent induction of new bone formation. It appears that the initiation of osteogenesis was delayed in the BMG group in our study but it was accelerated over time. 
In the $\mathrm{OCP} / \mathrm{BMG}$ group in the current study, a significant inflammatory reaction was noted in the first week following implantation. Mesenchymal cells had differentiated into osteoblast-like cells and covered the defect surface in a regular and orderly fashion. Osteoinduction in this group was greater at the margins compared to the center of defects in the second week. In the third week, new bone formation significantly increased and the amount of BMG particles absorbed was higher than OCP particles. High osteogenesis in this group may be partly because of the delayed absorption of OCP particles, since they induce the release of BMPs from the surrounding tissues and particularly the traumatized bone [17]. Besides, studies similar to this one must be carried out using additional radiographic and histomorphological evaluations in order to provide the necessary tools to allow for qualitative and quantitative comparisons among these materials.

\section{Conclusion}

The present study showed that implantation of OCP and BMG particles alone and in combination enhanced the regeneration of bone defects. Combination of OCP/BMG had greater potential for inducing osteoblastic differentiation and new bone formation compared to the application of OCP or BMG per se. It is probable or assumable that using a combination of osteoinductive and osteoconductive materials may be clinically beneficial to enhance regeneration of large bone defects.

\section{Acknowledgments}

The authors would like to thank the personnel of the animal research institute at the Zahedan University of Medical Sciences.

\section{References:}

1 - Amini AR, Laurencin CT, Nukavarapu SP. Bone tissue engineering: recent advances and challenges. Crit Rev Biomed Eng. 2012;40(5):363-408. 
2 - Canter HI, Vargel I, Marili ME. Reconstruction of mandibular defects using autografts combined with demineralized bone matrix and cancellous allograft. J Craniofac Surg. 2007;18(1):95-100

3 - Yan WQ, Oka M, Nakamura T. Bone bonding in bioactive glass ceramics combined with bone matrix gelatin. J Biomed Mater Res. 1998;42(2): 258-265.

4 - Lee FY, Storer S, Hazan EJ, Gebhardt MC, Mankin HJ. Repair of bone allograft fracture using bone morphogenetic protein-2. Clin Orthop Relat Res. 2002;397:119-126.

5 - Ashby ER, George H, Kenji Ishida. Evaluation of a novel osteogenic factor, Bone Cell stimulating substance in a rabbit cranial defect model. Plast Reconstr Surg. 1996;98(3):420-426.

6 - Combes C, Rey C. Amorphous calcium phosphates: Synthesis, Properties and uses in biomaterials. Acta Biomater. 2010;6(9): 3362-3378.

7 - Moseke C, Gbureck U. Tetracalcium phosphate: synthesis, properties and biomedical applications. Acta Biomater. 2010;6(10):3815-3823.

8 - Sun JS, Tsung YH, Liao C, Liu HC, Hang YS, Lin FH. The effect of calcium phosphate particles on the growth of osteoblasts. J Biomed mater Res. 1997;37(3):324-334.

9 - Kurashina K, Kurita H, Hirano M, Kotani A, Klein CP, Groot K. Invivo Study of calcium phosphate cements: Implantation of an alpha- Tricalcium phosphate/ dicalcium phosphate/ dicalcium phosphate dibasic/ Tetra calcium phosphate monoxide cement paste. Biomaterials 1997;18(7):539-543.

10 - Alghamdi HS, Junker R, Bronkhorst EM, Jansen JA. Regeneration related to calcium phosphate-coated implants in osteoporotic animal models: a meta- analysis. Tissue Eng Part B Rev. 2012;18(5):383-395.

11 - Araujo MV, Mendes VC, Chattopadhyay P, Davies JE. Low- Temperature particulate calcium phosphates for bone regeneration. Clin Oral Implants Res. 2010;21(6):632-641.

12 - Markovic M, Chow L. An octacalcium phosphate forming cement. J Res Nati Inst Stand Technol. 2010;115(4):257-265.

13 - Brandt J, Henning S, Michler G, Hein W, Berrstein A, Schulz M. Nanocrystaline hydroxyapatite for bone repair: an animal study. J mater Sci Mater Med. 2010;21(1):283-294.

14 - Suzuki O, Imaizumi H, Kamakura S, Katagiri T. Bone Regeneration by synthetic octacalcium phosphate and its role in biologic mineralization Curr Med chem. 2008;15(3):305313.

15 - Suzuki O. Octacalcium phosphate (OCP)- based bone substitue materials. Jap Dent Science Review. 2013;49:58-71.

16 - Kamakura S, Sasano Y, Homma H, Suzuki O, Kagayama M, Motegi K. Implantation of octacalcium phosphate(OCP) in rat skull defects enhances bone repair. J Dent Res.

1999;78(11):1682-1687.

17 - Sargolzaei aval F., Sobhani A., Arab MR., Sarani SA., Heydari MH. The efficacy of implant of octacalcium phosphate $(\mathrm{OCP})$ in combination with bone matrix gelatin $(\mathrm{BMG})$ on bone regeneration in skull defects in rat. Iran J. Med. Sci. 2004; 29(3):124-129. 
18 - Kamakura SH., Sasano Y., Nakajo S., Shimizu T., Suzuki O., et al. Implantation of octacalcium phosphate combined with transforming growth factor- $\beta 1$ Enhances bone repair as well as resorption of the implant in rat skull defects. J. Biomed. Mater. Res. 2001; 57:175-182.

19 - Ashby ER., George H., Kenji Ishida. Evaluation of a novel osteogenic factor, Bone Cell stimulating substance in a rabbit cranial defect model. Plast Reconstr Surg. 1996;98(3):420-426.

20 - Kim J, Lee KW, Ahn JH, Kim JY, Lee TY, Choi B. osteoinductivity depends on the ratio of demineralized bone matrix to a cellular dermal matrix in defects in Rat skulls. Tissue Eng. 2013;10(5):246-251.

21 - Lee FY, Storer S, Hazan EJ, Gebhardt MC, Mankin HJ. Repair of bone allograft fracture using bone morphogenetic protein-2. Clin Orthop Relat Res. 2002;397:119-126.

22 - khoshzaban A, Mehrzad S, Tavakoli V, Keshel SH, Behrouzi GR, Bashtar M. The comparative effectiveness of demineralized bone matrix, beta- tricalcium phosphate, and bovinederived anorganic bone matrix on inflammation and bone formation using a paired calvarial defect model in rats. Clin Cosmet Investing Dent. 2011;29(3):69-78.

23 - Lyon T, Scheele W, Bhandari M, Koval KJ, Sanchez EG, Christensen J, Valentin A, Huard F. Efficacy and safety of recombinant human bone morphogenetic protein- $2 /$ calcium phosphate matrix for closed tibial diaphyseal fracture: a double- blind, randomized, controlled phase-II/III trial. J Bone Joint Surg Am. 2013;95(23):2088-2096.

24 - Legeros RZ. Preparation of octacalcium phosphate(OCP):A direct fast method. Calcif Tissue Int. 1985;37:194-197.

25 - Suzuki O, Nakamura M, Miyasaka Y, Kagayama M, Sakurai M. Bone formation on synthetic precursors of hydroxyapatite. Tohoku J Exp Med. 1991;164(1):37-50.

26 - Suzuki O, Nakaura M, Miyasaka Y, Kagayama M, Sakurai M. Maclurapomifera agglutininbinding glycoconjugates on converted apatite from synthetic octacalcium phosphate implanted into subperiosteal region of mouse calvarial. Bone and mineral 1993; 20(2):151-66.

27 - Suzuki O, Yagishita H, Amano T, Aoba T. Reversible structural changes of octacalcium phosphate and labile acid phosphate. J. Dent Res 1995; 74: 1764- 1769.

28 - Urist MR, Iwata H. Bone morphogenesis in implants of insoluble bone gelatin. Proc Natl Amad Sci. 1973;70:3511-3515.

39 - Kamakura S, Sasano Y, Nakamura M, Suzuki O, Ohki H, Kagayama M, Motegi K. Initiation of alveolar ridge augmentation in the rat mandibular by subperiosteal implantation of octacalcium phosphate. Arch Oral Bio. 1996;41(11):1029-1038.

30 - Sasano Y, Kamakura S, Nakamura M, Suzuki O, Mizoguchi I, Kagayama M. Subperiosteal implantation of octacalcium phosphate stimulates both chondrogenesis and osteogenesis in the tibia but only osteogenesis in the parietal bone of a rat. Anat Rec. 1995;242(1):40-46.

31 - Mulliken JB, Glowacki J. Induced osteogenesis for repair and construction in the craniofacial region. Plast Reconst Surg 1980;65(5):553-559.

32 - Wang J, Glimcher MJ, Mah J, Zhou HY,Salih E. Expression of bone microsomal casein kinase II, bone sialoprotein and osteopontin during the repair of calvarial defects. Bone.

1998;22(6):621-628. 


\section{Figures:}
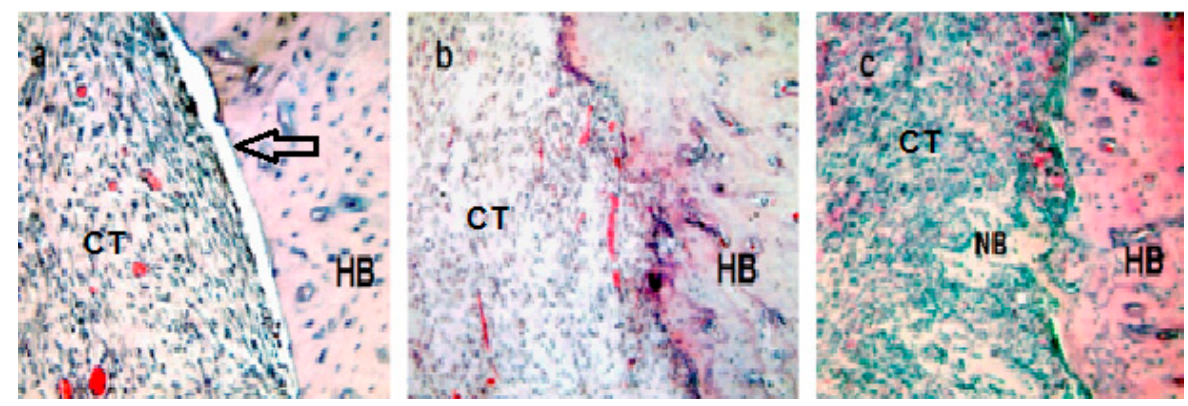

Figure 1. Histological micrograph of the control group at first week (a), second week (b) and third week (c) post-implantation. CT: connective tissue. HB: host bone. NB: newly formed bone.

Defect margin (long Arrow).H\&E staining, magnification=20×(a), 10× (b, c).

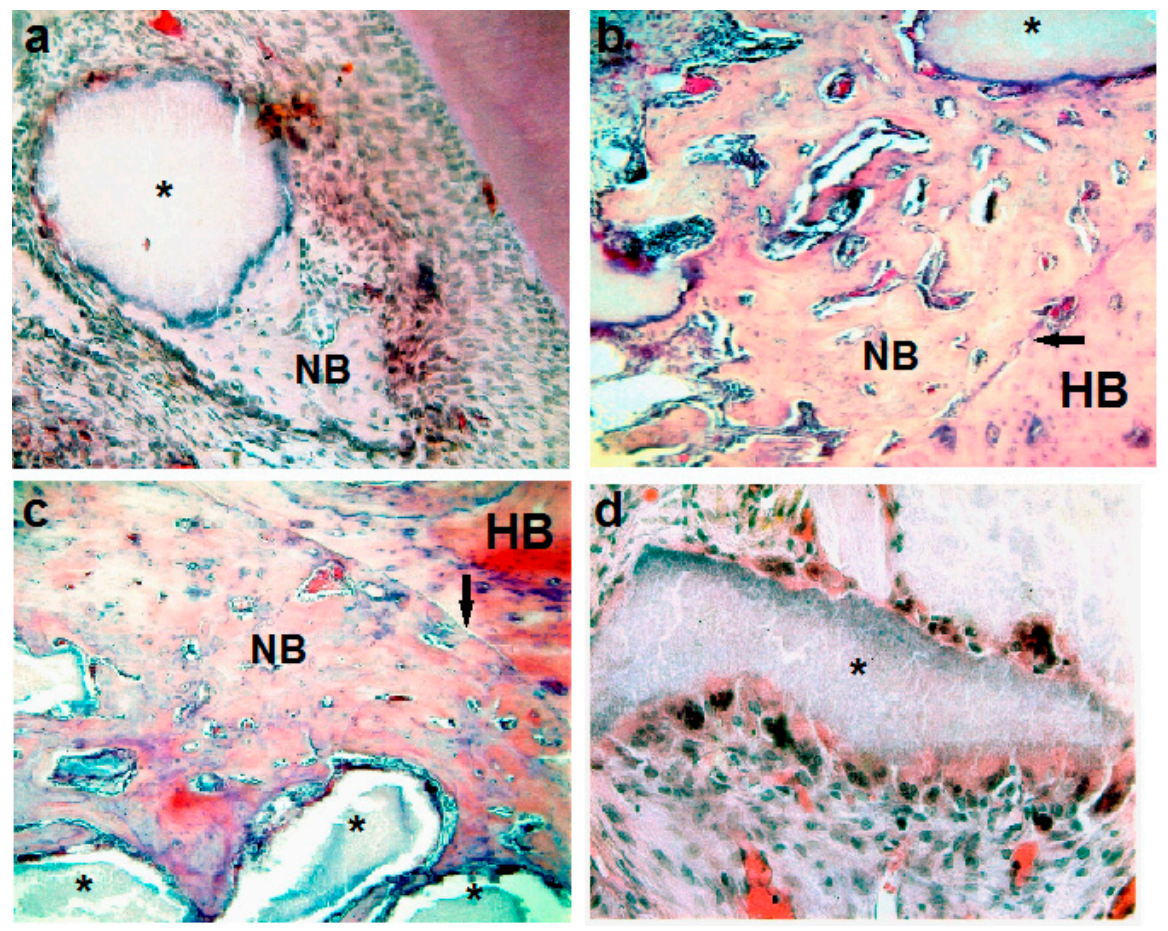

Figure 2. Histological micrograph of the OCP group at first week (a), second week (b) and third week(c, d) post-implantation. OCP particle (*). HB: host bone. NB: newly formed bone. Defect margin (long arrow). ). Multinuclear osteoclasts (short arrow). H\&E staining, magnification=20× (a), $10 \times(b, c), 40 \times(d)$. 


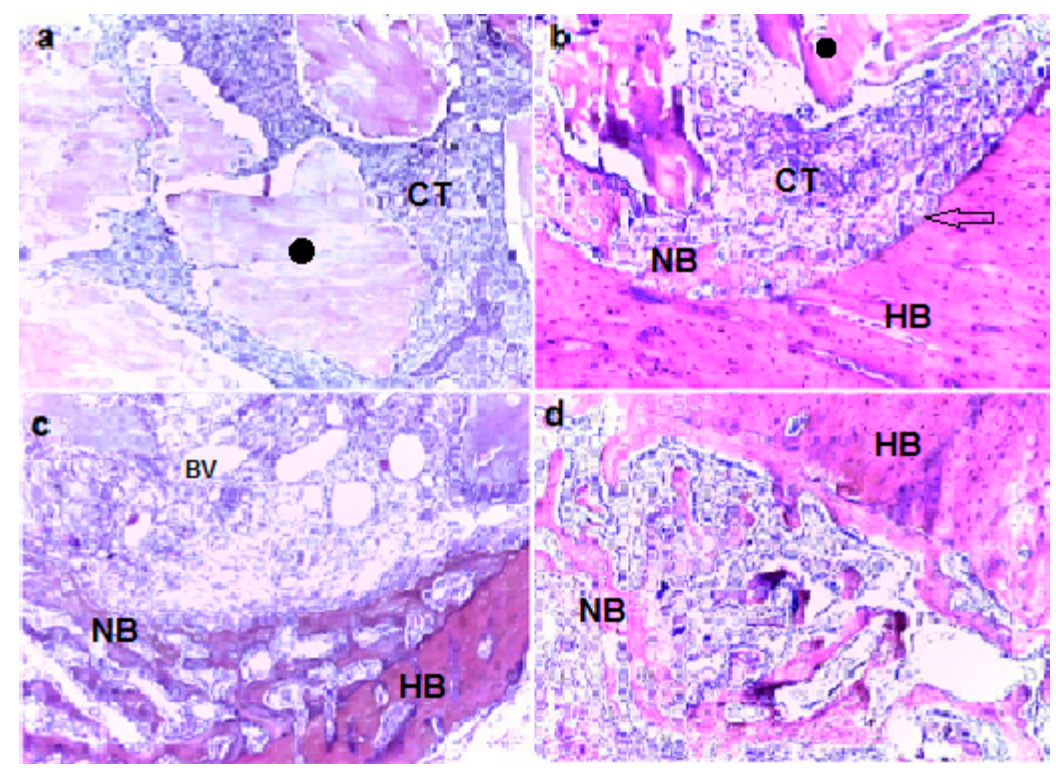

Figure 3. Histological micrograph of the BMG group at first week $(a, b)$, second week(c) and third week (d) post-implantation. BMG particle $(\bullet)$. CT: connective tissue. BV: blood vessels... HB: host bone. NB: newly formed bone. Defect margin (long arrow). H\&E staining, magnification $=10 \times(a, b, c, d)$.

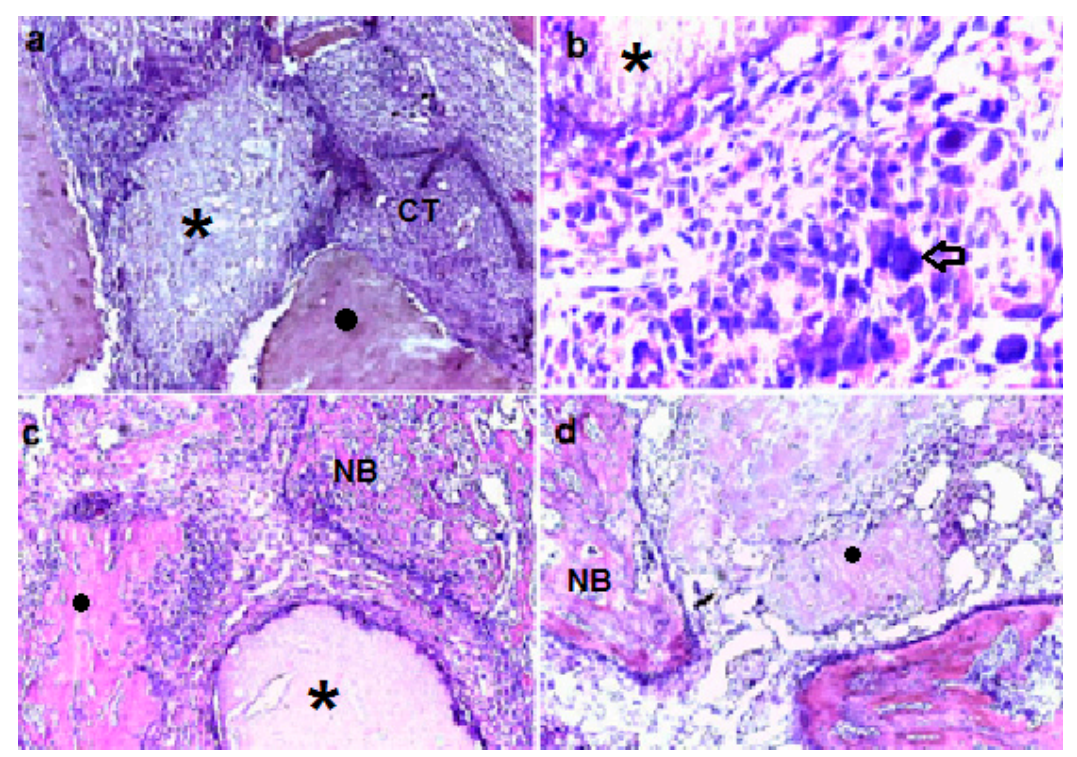

Figure 4. Histological micrograph of the OCP/BMG group at first week (a), second week (b , c) and third week (d) post-implantation. BMG particle $(\bullet)$. OCP particle $(*)$. CT: connective tissue.

BV: blood vessels. NB: newly formed bone. Multinuclear osteoclasts (short arrow). H\&E staining, magnification $=10 \times(\mathrm{a}, \mathrm{c}, \mathrm{d}), 40 \times(\mathrm{b})$. 